\title{
Additive representations on rank-ordered sets
}

\section{The topological approach}

\author{
Peter Wakker* \\ University of Nijmegen (NICI), Nijmegen, Netherlands
}

Submitted March 1990, accepted July 1991

Additive representation theory on subsets of Cartesian products has characteristics different from additive representation theory on full Cartesian products. This paper describes the difficulties that can arise on subsets. These difficulties have been underestimated in the literature. For the special case of rank-ordered subsets of Cartesian products the paper obtains characterizations of additive representations. These results can be applied in the modern rank-dependent approaches to decision making under risk/uncertainty, and to generalizations of the Gini index in the measurement of inequality.

\section{Introduction}

This paper studics additive representation theory on subsets of Cartesian products. Interest in this topic has increased during the last decade because of new developments in decision making under risk and uncertainty, and because of new developments in the measurement of inequality. This paper considers the topological approach, i.e., it makes use of continuity assumptions. Wakker (1991) has given results for the algebraic approach, where solvability and an Archimedean axiom are used instead of continuity. The present paper builds on the latter one. A comparison of the algebraic and the topological approach has been given in Wakker (1988), and in Luce et al. (1990, see p. 49, 1. 10).

Traditional expected utility theory was characterized mainly by independence' axioms. Dissatisfaction with expected utility has led to weakenings of independence that require independence to hold only within certain subdomains. Special cases of this general procedure for weakening independence

Correspondence to: Peter Wakker, University of Nijmegen (NICI), Psychological Laboratory, P.O. Box 9104, 6500 HE Nijmegen, The Netherlands.

*The research has been made possible by a fellowship of the Royal Netherland Academy of Arts and Sciences, and a fellowship of the Netherlands Organization for Scientific Research. Discussions with Alain Chateauneuf initiated this research. An anonymous referee gave helpful comments. 
include Chew (1989), who requires independence to hold only within equivalence classes, and the 'twins' Jaffray (1988) and Gilboa (1988), who require independence to hold only, among others, when minimal outcomes are identical. In Becker and Sarin's (1987) 'lottery-dependent expected utility' the sets of lotterics within which independence should hold arc characterized by having the same (lottery-dependent) utility function. The present paper has been motivated by the rank-dependent theories initiated by Quiggin (1982) and Schmeidler (1989, first version 1982), which require independence to hold only on 'comonotonic' subsets. The literature and history of rank-dependent theories have been discussed in Wakker (1991). After completion of that paper we found Birnbaum (1974, p. 559) and Birnbaum and Sutton (1990). These papers deal with 'configural weight theory', which is similar to rank-dependent utility in that decision weights are also allowed to depend on ranking.

Section 2.3 discusses several papers dealing with additive representations on subsets of Cartesian products. In addition let us mention here that the case of additive functions, linear with respect to a mixture operation, has been dealt with by Fishburn (1976). The present paper will not assume linearity with respect to mixing. Necessary and sufficient conditions for general additive representability on arbitrary countable subsets of Cartesian products were provided by Jaffray (1974b) for two dimensions, and Jaffray (1974a) for arbitrary finite dimensions. We study the special case of additive representations that are continuous with respect to a connected topology. Euclidean spaces are, of course, the most important example of this type. In two dimensions, Blaschke and Bol (1938, Chapter 1) derived this representation for open, simply connected domains in which all equivalence classes and all intersections of the domain with hyperplanes perpendicular to an axis are connected. Recently Segal (1990) showed how to extend these results to finite dimensions greater/equal three, and to open domains within Euclidean spaces that are connected instead of simply connected.

It has occasionally been thought that additive representation theory on subsets of Cartesian products does not differ from additive representation theory on full Cartesian products, when the subsets have interiors that are 'full-dimensional' and connected. A first counter-example, refuting several conjectures in the literature, was given in Wakker (1989b, Remark III.7.8). It is illustrated and modified in fig. 2 below. Indeed, Krantz et al. (1971, p. 276, lines 6/7) had already suggested that the subject is nontrivial. We shall observe the following phenomenona, typical for subsets of Cartesian products in the topological approach:

(1) [See Example 3.8.] One reason that the traditional axioms no longer imply additive representability is that, looscly speaking, an additive representation may be 'driven to infinity' at maximal alternatives, and to minus infinity at minimal alternatives. 
(2) [See Examples 3.9 and 3.10.] Continuity of $\geqslant$ on a subset $X$ of the Cartesian product $\mathscr{C}^{n}$ no longer has to imply continuity of the associated binary relation $\succcurlyeq^{\prime}$ on $\mathscr{C}$.

(3) [See fig. 2 below, or Remark III.7.8 of Wakker (1989b) with restriction to interiors.] In general, even if the subset is full-dimensional, connected and open, the traditional axioms of additive representation theory still need not imply additive representability.

(4) [See figs. 1 and 2 below, or Remark III.7.8 of Wakker (1989b).] 'Local additive representability' (for the definition see Claim 2.1 below) no longer implies ('global') additive representability.

We shall show that on rank-ordered subsets of Cartesian products additive representation theory essentially remains valid, as long as maximal and minimal alternatives are excluded. Loosely speaking, rank-ordered subsets provide sufficient overlap between different local additive representations to make these fit together into one global additive representation. The results of this paper will be used in Chew and Wakker (1991) to characterize the preference relations on full Cartesian products that satisfy 'comonotonic independence'. Comonotonic independence requires that the usual additive representation axioms hold only within rank-ordered subsets.

This paper is organized as follows. In section 2 we discuss some common errors that have caused many misunderstandings in the literature, and might be feared to continue doing so. Section 2.1 describes them without yet criticizing, giving the reader the opportunity to find the mistakes by him- or herself. Section 2.2 points out the mistakes, and section 2.3 discusses the literature. Section 3 gives the main results of this paper. It can be read independently of the other sections. Section 3.5 gives examples that show peculiarities of extreme alternatives in rank-ordered subsets. Proofs are given in section 4. Section 5 concludes.

\section{Common misconceptions}

This section discusses some erroneous reasonings. Their alleged correctness has been accepted in the literature as a kind of common knowledge. To prevent future misunderstandings we have decided to present these reasonings in detail, see section 2.1 . The reader is invited to find the mistakes by him- or herself, prior to reading section 2.2 where they are exposed.

\subsection{Common beliefs}

For simplicity we assume in this subsection that the domain of the preference relation is (a subset of) $\mathbb{R}^{n}$, and that the preference relation is a continuous and increasing weak order. For consistency of terminology, elements of $\mathbb{R}^{n}$ are called alternatives. 
Claim 2.1 [From local to global additive representation]. If for every alternative in $\mathbb{R}^{n}$ there is a neighborhood on which there is an additive representation for the preference relation (local additive representability) then there exists a ('global') additive representation.

Alleged Proof. Let us repeat that by the well-known uniqueness results additive representations are unique up to scale and location. The neighborhoods may and will be assumed to be open rectangles. We use as-if twodimensional terminology.

Step 1. Take any starting alternative. Take the open rectangle around the starting alternative, and the additive representation on this rectangle.

Step 2. Next take any second rectangle that intersects the first, for example one around a boundary point of the first. Take the additive representation on the second rectangle. On the intersection of the first and second rectangle (that intersection is itself a rectangle) the two additive representations represent the same preference relation. Hence by standard uniqueness results we can change scale and location of the additive representation on the second rectangle so that it coincides with the additive representation of the first rectangle on common domain; so we do. We have obtained an additive representation on the union of two rectangles.

Step $k$. Suppose $k-1$ rectangles have been covered. Take any rectangle that has not yet been covered and that intersects the area already covered, and the additive function $V^{k}$ on that new rectangle. On the intersection with the area already covered it represents the same preference relation as the additive function $V$ constructed so far. Hence by standard uniqueness results we can choose scale and location so that $V^{k}$ coincides with $V$ on common domain.

By the lemma of Zorn, or countable induction and topological separability, this process will stop only if the entire domain $\mathbb{R}^{n}$ has been covered. A global additive representation has been obtained.

Claim 2.2 [Additive representation on subsets]. The usual characterization results of Debreu (1960) and Gorman (1968) also apply to subsets of $\mathbb{R}^{n}$ if these subsets are connected and have nonempty interior (and only contain boundary points that are limits of interior points).

Alleged Proof. For every interior point there is an open rectangle around the point contained within the set, on which by the traditional results 
additive representability is ensured. I.e., on the interior we have local additive representability. Then, by a reasoning completely analogous to the Alleged Proof of Claim 2.1, we get an additive representation on the interior. Extension to the boundary is by continuity.

The Alleged Proof of Claim 2.2, building on the Alleged Proof of Claim 2.1, in fact also gives an Alleged Proof for the following Claim 2.3, which strengthens both Claim 2.1 and Claim 2.2.

Claim 2.3. Claim 2.1 also applies to the subsets as described in Claim 2.2.

\subsection{Flaws in the alleged proofs}

Flaws in the Alleged Proof of Claim 2.1. We distinguish four flaws in the Alleged Proof of Claim 2.1, of which the fourth flaw is generated by the first three.

The first flaw occurs at Step 2. There we have constructed an additive function on a union of two rectangles, that is representing on each of the rectangles. After a little reflection the reader will see that this does not mean that the additive function is representing on the union of the two rectangles. In general, if a function on a union of two sets is representing on each of the two sets, it need not be representing on the union. The additive function may compare incorrectly an element from the first rectangle that is not contained in the second, with an element from the second rectangle that is not contained in the first. See fig. 2 , where $W$ is representing both on the rectangle $E_{1} \cup E_{2}$, and on the rectangle $E_{1} \cup E_{3}$, but not on their union.

The second flaw does not occur at the first or second step, but only at later steps. Suppose we have constructed an additive representation on the first $k-1$ rectangles, and want to include the $k$ th rectangle, intersecting the area already covered. On the intersection the two additive functions represent the same preference relation. This does not ensure that they therefore differ only by scale and location. That has been established in the literature only for the special case where the intersection is a connected Cartesian product, and can readily be extended to any connected set. The intersection is not necessarily a connected set though. As a general example, think of the case where the intersection consists of two disjoint parts $A$ and $B$ that share no coordinates; suppose all elements of $A$ are strictly preferred to those of $B$. An additive representation is given on the union of the two, it is positive on $A$, and negative on $B$. Then we can multiply the function by two on $A$ and leave it unchanged on $B$, to outain an alternative additive representation on the union. It has not been obtained from the original one in the usual way. 


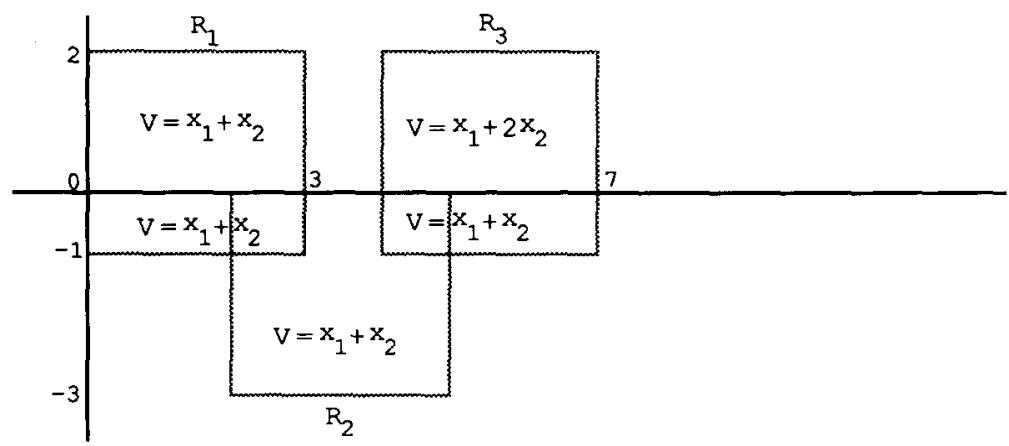

Fig. 1 [A function that is additive on two sets need not be additive on the union of these two sets]. Suppose, in $\mathbb{R}^{2}, R_{1}=[0,3] \times[-1,2], R_{2}=[2,5] \times[-3,0], R_{3}=[4,7] \times[-1,2], V$ is $x_{1}+2 x_{2}$ on the subset $[4,7] \times[0,2]$ of $R_{3}, V$ is $x_{1}+x_{2}$ on the remainder of $R_{1} \cup R_{2} \cup R_{3} . V$ is additive on $R_{1} \cup R_{2}$, as well as on $R_{2} \cup R_{3}$. But $V$ is not additive on the union $R_{1} \cup R_{2} \cup R_{3}$ because then contradictory requirements would result for the second-coordinate function on $[0,2]$ : should it be the identity or two times the identity? The binary relation represented by $V$ is locally additively representable, but not globally.

The third flaw also does not occur at the first or second step, but only at later steps. Suppose again we have constructed an additive representation on the first $k-1$ rectangles, and want to include the $k$ th rectangle, intersecting the area already covered. Suppose we can escape from the second flaw and can 'fit together' the representations, i.e., obtain one function on the union of these $k$ rectangles that is additive and representing on the union of the first $k-1$ rectangles, and on the $k$ th rectangle. In the first flaw above we already saw that this one function may not be representing on the union of the $k$ rectangles; in addition, the function may neither be additive. In general, a function that is additive on each of two sets need not be additive on their union. See fig. 1.

There is a fourth flaw, generated by the first and third. To wit, let us look at the $k$ th step. It was claimed that the additive representation on the $k$ th rectangle would on the intersection with the area already covered represent the same preference relation as the additive representation already constructed. This is not necessarily true because of the first and third flaws. The function as already constructed does not have to be representing or additive.

Flaws in the Alleged Proof of Claim 2.2. Firstly of course the Alleged Proof builds on the Alleged Proof of Claim 2.1, thus shares its flaws. In addition, there is a fifth flaw: additive representation on the boundary may go wrong. Loosely speaking, the additive representation may be 'driven to $\infty$ ' at maximal alternatives, and to $-\infty$ at minimal alternatives, even if there exists a bounded real-valued continuous (nonadditive) representation on the set. See Example 3.8 . 
An actual counterexample against Claims 2.2 and 2.3 has been provided in Remark III.7.8 of Wakker (1989b). A modified version is given in fig. 2.

One reason for the persistence of the erroneous beliefs in these claims and their alleged proofs may be their mutual support. If someone believes the claims to be true, then a momentary doubt related to the flaws in the Alleged Proof of Claim 2.1 will be silenced by the belief in Claim 2.2. Conversely, momentary doubt about Claim 2.2 will be silenced by the Alleged Proof of Claim 2.1, which also gives Claim 2.3, implying Claim 2.2.

\subsection{A discussion of literature}

The Alleged Proof, or small variations, can be found in Green and Jullien (1988, Lemma in Appendix), Ebert (1988a, proof of Theorem 1; and 1988b, top of p. 220; both presentations concise), as well as in some other places in the decision literature. For functional equations it can be found in Radó (1959, proofs of Theorems 1 and 2) and in Hosszù (1964, Section 3; note that the domain in fig. 2 is simply connected). Radó (1959, Theorems 1 and 2) formulates Claims 2.2 and 2.3 for arbitrary domains. Applications of functional equations have more often been inaccurate concerning domains. Von Stengel (1990) mentions problems in Gorman's (1968) application of Aczél (1966, p. 311/312 Theorem 1 and Corollary 1), and presents corrections and generalizations. In the decision literature a version of Claim 2.2 is found in Quiggin (1989, Proposition 4). See also Kahneman and Tversky (1979, Appendix; since the probabilities sum to 1, the domain there is not a full Cartesian product). Other derivations, while basically correct, have dealt with the problems described above in very concise and implicit manners. Let us, given its importance, discuss in detail Debreu (1960, Theorem 3). Below fig. 1 ('Actually, ... type') of that paper, Claim 2.1 is made for dimension 2. Debreu (1990) referred to Blaschke and Bol (1938), where it is indeed found in Exercise 8 of Section I.2, p. 22, be it with little elaboration. This result is essentially used in Debreu (1960, proof of Theorem 3), when for global additive representability on the two-dimensional sets denoted $T_{1} \times T_{2}$ only local ('in the small') additive representability, on neighborhoods denoted $U$, has been established. Again, for extension of the additive representation from sets $T_{1} \times \cdots \times T_{k-1}$ to sets $T_{1} \times \cdots \times T_{k}$ there, only local additive representability has been established, on neighborhoods denoted $V$. Because the dimensions $1, \ldots, k-1$ can be taken together, this can be reformulated as a two-dimensional problem, and can (first within each compact subset) be derived from the result in Blaschke and Bol (1938). So again it can be justified; sometimes Debreu has been criticized for these two steps. Note however that the domain (i.e., the domain of the function denoted $g_{k}$ ) there is not necessarily a full Cartesian product; this can be seen to be no serious problem, by the reformulation as mentioned above and Blaschke and Bol 

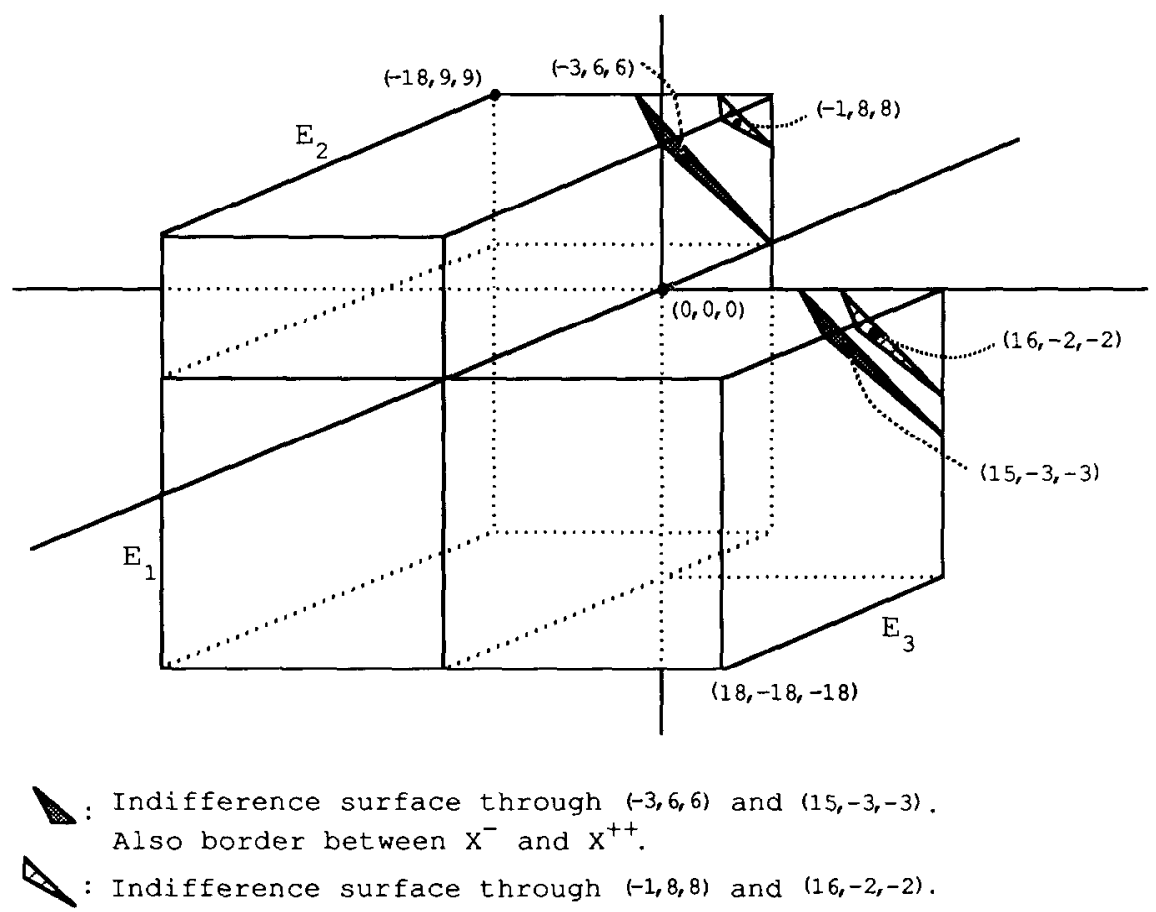

Fig. 2 [Counterexample to additive representation on subsets of Cartesian products, also showing that local additive representability need not imply global additive representability]. $E_{1}:=[-18,0] \times[-18,9] \times[-18,0], E_{2}:=[-18,0] \times[-18,9] \times[0,9], E_{3}:=[0,18] \times[-18,0] \times$ $[18,0] . \quad X:=E_{1} \cup E_{2} \cup E_{3} . \quad W: X \rightarrow \mathbb{R}$ is defined by $W:\left(x_{1}, x_{2}, x_{3}\right) \mapsto x_{1}+x_{2}+x_{3}-9$. $X^{-}:=\left\{\left(x_{1}, x_{2}, x_{3}\right) \in X: W\left(x_{1}, x_{2}, x_{3}\right) \leqq 0\right\}, X^{++}:=\left\{\left(x_{1}, x_{2}, x_{3}\right) \in X: W\left(x_{1}, x_{2}, x_{3}\right)>0\right\}$. The function $V$ is defined as $W / 2$ on $E_{2} \cap X^{++}$, as $W$ on the remainder of $X$. The binary relation $\geqslant$ is represented by $V$.

Note that $W$ represents $\geqslant$ not only on $E_{1} \cup E_{2}$, but also on $E_{1} \cup E_{3}$. Still $W$ does not represent $\geqslant$ on the entire $X$; it compares incorrectly pairs of elements $x, y$ with $x \in X^{++} \cap E_{2}$ and $y \in X^{++} \cap E_{3}$. To wit: $W(-1,8,8)=6>3=W(16,-2,-2)$ but $V(-1,8,8)=3=$ $V(16,-2,-2)$ so that $(-1,8,8) \sim(16,-2,-2)$.

For every element of $X$ there exists a neighborhood on which the preference relation has an additive representation, and on which $V$ is a strictly increasing transform of that additive representation: $W$ can always be taken for that additive representation, and $X^{-}$or $E_{2}$ or $E_{3}$ for the neighborhood. Still, the representing function $V$ is not a transform of any additive function. For suppose it were; contradiction will follow. The additive function would be of the form $\phi \circ W$ on $E_{1} \cup E_{2}$, and of the form $\phi^{\prime} \circ W$ on $E_{1} \cup E_{3}$, for positive affine $\phi, \phi^{\prime}$. From $E_{1}$ we infer that $\phi=\phi^{\prime}$. However, then $V$ would be a strictly increasing transform of $W$ on $X$. This is impossible because $W$ is not representing on the entire $X$.

The preference relation satisfies all usual conditions for additive representability, only the domain is not a full Cartesian product. To wit, $\geqslant$ is transitive, complete, and continuous. Its domain $X$ is topologically connected (even simply connected). Also $\geqslant$ satisfies CI. This follows mainly from the fact that the two separate parts of $X^{++}$have no coordinates in common. This also shows that the meaning of $\mathrm{CI}$ [and, more generally, in the terminology of Krantz et al. (1971) of cancellation axioms] is sensitive to the shape of the domain. 
(1938). Debreu does not discuss the extension of the representation to the boundary of the domain, but on full Cartesian products this extension does not pose any problem. Also in Fishburn (1970, p. 75/76) some problems have not been elaborated.

Positive references are Doignon and Falmagne (1974) for difference measurement, and Wakker (1986, Chapter VI; 1989a; 1989b, Chapter VI), Miyamoto (1988, for $n=2$ ), and Nakamura (1990) for Choquet expected utility, i.e., expected utility on rank-ordered sets. [Lemma 2 in Nakamura (1990), is subject to the 'driven to infinity' problem, but this is no serious problem.] To the best of our knowledge, all these authors obtained the first versions of their results independently. They avoided the flaws indicated above by employing, in an early stage of the proof, the proportionality of the additive value functions that is available in their set-ups.

The correctness of Claim 2.1 has been an open problem for me ever since my first reading of the claim in Debreu (1960, below fig. 1). This, together with the recent developments in decision theory, has triggered my investigation. Only when Debreu (1990) pointed out that his referring to Blaschke and Bol (1938) also covered this claim, and when I understood from Fishburn (1970, fig. 5.7 and p. 74, Step 5) that it sufficed to prove the result on compact sets, did I find a proof, in Wakker (1990). Obviously, this proof is more involved than the alleged proof as sketched above.

\section{The main results}

\subsection{Rank-ordered alternatives; extreme alternatives; the set $X$}

$\mathscr{C}$ is a nonempty connected topological space, with elements called outcomes. $\succcurlyeq^{\prime}$ is a weak order on $\mathscr{C}$, i.e., it is complete $\left(\forall \alpha, \beta \in \mathscr{C}: \alpha \geqslant{ }^{\prime} \beta\right.$ or $\beta \succcurlyeq^{\prime} \alpha$ ) and transitive. $\succ^{\prime}, \preccurlyeq{ }^{\prime},\left\langle^{\prime}, \sim^{\prime}\right.$ are as usual. We assume that $n \geqq 2$. An $n$-tuple $\left(x_{1}, \ldots, x_{n}\right) \in \mathscr{C}^{n}$ is rank-ordered if $x_{1} \geqslant^{\prime} x_{2} \geqslant^{\prime} \cdots \geqslant^{\prime} x_{n}$, and is then called a rank-ordered alternative. The set of rank-ordered alternatives is denoted as $\mathscr{C}_{r}^{n}$. Since non-rank-ordered alternatives will not be considered in this paper, we simply say alternative instead of rank-ordered alternative. The restriction to rank-ordered alternatives implies, in the terminology introduced by Schmeidler (1989), that all alternatives are 'comonotonic'. Thus 'coordinate independence' in our set-up will be identical to 'comonotonic coordinate independence'.

An outcome $\alpha$ is maximal if $\beta \succ^{\prime} \alpha$ for no outcome $\beta, \alpha$ is minimal if $\beta<^{\prime} \alpha$ for no outcome $\beta$. Note that a rank-ordered alternative with maximal $n$th coordinate must have all of its coordinates maximal, and a rank-ordered alternative with minimal first coordinate must have all of its coordinates minimal. We call such alternatives extreme. We shall see in this paper, primarily in section 3.5 , that complications and new phenomena can occur 
for extreme alternatives when the domain of the preference relation is not a full Cartesian product.

We consider a binary (preference) relation $\succcurlyeq$ on a set $X \subset \mathscr{C}_{r}^{n}$. Either $X$ will be the set of all (rank-ordered) alternatives, or it will be that set with the extreme alternatives excluded. Again $\succ, \preccurlyeq,<, \sim$ are as usual. Often a 'constant' alternative $(\alpha, \ldots, \alpha) \in X$ will be identified with the outcome $\alpha \in \mathscr{C}$. Transitivity and the monotonicity assumption of this paper will ensure that $\succcurlyeq^{\prime}$ and $\succcurlyeq$ are in agreement, i.e., for all $(\alpha, \ldots, \alpha),(\beta, \ldots, \beta) \in X$ : $(\alpha, \ldots, \alpha) \geqslant(\beta, \ldots, \beta) \Leftrightarrow \alpha \geqslant{ }^{\prime} \beta$.

\subsection{Intuitive conditions of additive representations}

Throughout, suppose $E \subset X$. Because we consider varying subsets of $\mathscr{C}^{n}$, it is useful to define the properties introduced below for subsets of Cartesian products. Whenever below $E=X$ ( $X$ the entire domain of $\geqslant$ ), we omit the phrase 'on $E$ '. $\geqslant$ is a weak order on $E$ if it is transitive on $E(\forall x, y, z \in E$ : $[x \geqslant y, y \geqslant z] \Rightarrow[x \geqslant z])$ and complete on $E(\forall x, y \in E: x \geqslant y$ or $y \geqslant x)$. A function $V$ represents $\geqslant$ on $E$ if $E$ is contained in the domain of $V, V$ 's range is $\mathbb{R}$, and $x \geqslant y \Leftrightarrow V(x) \geqq V(y)$. A function $V$ is additive on $E$ if $\forall x \in E$ : $V(x)=\sum_{j=1}^{n} V_{j}\left(x_{j}\right)$ for some functions $V_{1}, \ldots, V_{n}$ to $\mathbb{R}$. If an additive function represents $\geqslant$, then the $V_{j}$ 's are additive value functions. We sometimes say that an additive representing function is 'the sum' of the additive value functions. Given different domains this is a slight abuse of terminology. A function $V$ is cardinal (or an 'interval scale') if, loosely speaking, it 'can be replaced' by any function $\tau+\sigma V$ for real $\tau$ and positive $\sigma$. Additive value functions $\left(V_{j}\right)_{j=1}^{n}$ are jointly cardinal if they can be replaced by $\left(W_{j}\right)_{j=1}^{n}$ if and only if there exist real $\tau_{1}, \ldots, \tau_{n}$, and a positive $\sigma$, such that $\forall j: W_{j}=\tau_{j}+\sigma V_{j}$.

If in the above definitions the range of $V$ (and the $V_{j}^{\prime}$ s) is $\mathbb{R} \cup\{-\infty, \infty\}$ instead of $\mathbb{R}$, then $V$ is called an extended representation, respectively extended additive representation, and the $V_{j}$ 's are called extended additive value functions. Obviously an (additive) representation is an extended additive representation, and additive valuc functions arc cxtended additive value functions. $\mathbb{R} \cup\{-\infty, \infty\}$ is endowed with the usual addition and multiplication rules, with $\infty-\infty$ undefined. We write $x_{-i} \alpha$ for $x$ with $x_{i}$ replaced by $\alpha$, and, for $i \neq j, x_{-i, j} \alpha, \beta$ for $x$ with $x_{i}$ replaced by $\alpha, x_{j}$ replaced by $\beta$. If extended additive value functions exist on $E \subset X$ then $\geqslant$ is coordinate independent $(C I)$ on $E$, i.e., $x_{-i} \alpha \geqslant y_{-i} \alpha \Leftrightarrow x_{-i} \beta \geqslant y_{-i} \beta$ whenever all alternatives in question are contained in $E$. As shown in Wakker (1991), on rank-ordered sets $\mathrm{CI}$ implies that preferences are independent not only of one common coordinate, but of any set of common coordinates. If extended additive value functions exist for $\geqslant$ on $E \subset \mathscr{C}^{n}$ then $\geqslant$ satisfies generalized triple cancellation on $E \subset \mathscr{C}^{n}$, i.e., for all $x_{-i} \alpha, \ldots, w_{-i} \delta \in E$ : 


$$
\begin{array}{ll} 
& \begin{array}{l}
x_{-i} \alpha \leqslant y_{-i} \beta \quad \& \quad v_{-i} \alpha \geqslant w_{-i} \beta \\
\& \quad x_{-i} \gamma \geqslant y_{-i} \delta
\end{array} \\
\Rightarrow & \quad v_{-i} \gamma \geqslant w_{-i} \delta .
\end{array}
$$

Note that, for reflexive $\succcurlyeq$, this implies CI, by setting $\alpha=\beta, \gamma=\delta, x=y$. The binary relation $\geqslant$ satisfies monotonicity on $E$ if $\alpha \geqslant \beta \Leftrightarrow x_{-i} \alpha \geqslant x_{-i} \beta$ for all $x_{-i} \alpha, x_{-i} \beta \in E$. Analogously a function $V$ to $\mathbb{R}$ satisfies monotonicity on $E$ if $E$ is in the domain of $V$ and $\alpha \geqslant \beta \Leftrightarrow V\left(x_{-i} \alpha\right) \geqq V\left(x_{-i} \beta\right)$ for all $x_{-i} \alpha, x_{-i} \beta \in E$. Also we call, for $E_{j}=\mathscr{C}$, a function $V_{j}: E_{J} \rightarrow \mathbb{R}$ monotonic on $E_{J}$ if $E_{j}$ is in the domain of $V_{j}$ and $\forall \alpha, \beta \in E_{j}: \alpha \geqslant{ }^{\prime} \beta \Leftrightarrow V_{j}(\alpha) \geqq V_{j}(\beta)$.

\subsection{Technical conditions of additive representations}

In this section we give some technical conditions. They can be skipped by readers not interested in details. Most of these conditions are only used in proofs; the definition of the second-order Archimedean axiom is used only in Theorem 3.3(c) and its proof.

Coordinate $i$ is inessential on $E$ (with respect to $\geqslant$ ) if $\forall x, x_{-i} \alpha \in E: x \sim x_{-i} \alpha$. The opposite of inessential is essential. Next we define the Archimedean axiom. As a preparation we define a standard sequence; a standard sequence may be infinite or finite of any length. For coordinate $j$ we call $\alpha^{1}, \alpha^{2}, \ldots$ a standard sequence (on coordinate $j$ ) with respect to $\geqslant$ on $E \subset \mathscr{C}^{n}$, if there exist $x, i \neq j, v_{i} \nsucc w_{i} \in \mathscr{C}$ such that, for all $k=1,2, \ldots$, we have $x_{-i, j} v_{i}, \alpha^{k} \in E$, $x_{-i, j} w_{i}, \alpha^{k+1} \in E$, and

$$
x_{-i, j} v_{i}, \alpha^{k} \sim x_{-i, j} \mathbf{w}_{i}, \alpha^{k+1}
$$

A sequence $\alpha^{1}, \alpha^{2}, \ldots$ on coordinate $j$ is bounded on $E$ if there exist $\alpha^{\text {sup }}$ and $\alpha^{\text {inf }}$ such that $\alpha^{\text {sup }} \geqslant^{\prime} \alpha^{k} \geqslant^{\prime} \alpha^{\text {inf }}$ for all $k$, with $x_{j}=\alpha^{\text {inf }}, y_{j}=\alpha^{\text {sup }}$ for some $x, y \in E$ (the latter to ensure that $\alpha^{\text {sup }}, \alpha^{\text {inf }}$ are in the range of additive value functions, if these exist). The Archimedean axiom requires that every bounded standard sequence is finite. On rank-ordered sets a stronger notion of the Archimedean axiom will be needed. For coordinate $j$ we call $\alpha^{1}, \alpha^{2}, \ldots$ a second-order standard sequence (on coordinate $j$ ) with respect to $E \subset \mathscr{C}^{n}$, if for every $k$ there exists a standard sequence $\beta^{1}, \beta^{2}, \ldots$ on coordinate $j$ with respect to $\geqslant$ on $E$, such that, for some $m, m^{\prime}, l \in \mathbb{N}, \alpha^{k} \sim^{\prime} \beta^{m}, \alpha^{k+1} \sim^{\prime} \beta^{m+l}$, and $\alpha^{k+2} \sim^{\prime} \beta^{m+2 l}$. The second-order Archimedean axiom requires that every bounded second-order standard sequence is finite. Since any standard sequence is a second-order standard sequence, the second-order Archimedean axiom implies the usual one. Again it is necessary for additive representa- 
bility: the $V_{j}$-differences of subsequent outcomes in a second-order standard sequence are constant. The condition is somewhat less unattractive and, by itself, less restrictive than the strong Archimedean axiom as needed in the algebraic approach in Wakker (1991).

A comprehensive presentation of elementary topological definitions, and further definitions, is given in Wakker (1989b, Section 0.1). Readers not familiar with general topology may simply assume that $\mathscr{C}=\mathbb{R}$, and that preferences are increasing $\left(\alpha>\beta \Rightarrow x_{-i} \alpha>x_{-i} \beta\right)$; then all topological assumptions below are satisfied. Without further mention we assume throughout that $X$, as well as any $E \subset X$, are endowed with the restriction of the product topology on $\mathscr{C}^{n}$. We call $\geqslant$ continuous on $E$ if for all $y \in E$ the sets $\{x \in E$ : $x \geqslant y\}$ and $\{x \in E: x \preccurlyeq y\}$ are closed. Analogously, $\geqslant$ on $\mathscr{C}$ is continuous if for all $\alpha \in \mathscr{C}$ the sets $\left\{\beta \in \mathscr{C}: \beta \succcurlyeq^{\prime} \alpha\right\}$ and $\left\{\beta \in \mathscr{C}: \beta \preccurlyeq^{\prime} \alpha\right\}$ are closed. In general, continuity of $\geqslant$, or continuity of additive value functions, does not imply continuity of $\succcurlyeq^{\prime}$ on $\mathscr{C}$. Continuity of $\succcurlyeq^{\prime}$ is, however, needed in the main results, hence will be presupposed in the Structural Assumption 3.1.

\subsection{The main theorems}

This subsection gives the main theorems. These adapt the results of Wakker (1991) to the topological approach. For the sake of easy reference and self-containedness we shall spell out several straighforward corollaries. Let us repeat the assumptions of our set-up:

Assumption 3.1 [Structural Assumption]. $\mathscr{C}$ is a nonempty connected topological space, $n \geqq 2$, and subsets of $\mathscr{C}^{n}$ are endowed with the restriction of the product topology. $\geqslant$ is a binary relation on a set $X \subset \mathscr{C}_{r}^{n}$ of rank-ordered alternatives. Rank-ordering is with respect to a continuous weak order $\geqslant$ ' on $\mathscr{C}$ 'agreeing' with the binary relation $\geqslant$ on $X$ restricted to constant alternatives.

The assumption of continuity of $\geqslant$ can often be deleted, for instance for $n \geqq 3$ in Theorem 3.2. See Proposition 3.11. In the topological approach it is more convenient to formulate conditions for additive value functions, than for their sum (the additive representation) as was done in Wakker (1991).

Theorem 3.2. Suppose the Structural Assumption 3.1 holds with $X-\mathscr{C}_{r}^{n}$. Then the following two statements are equivalent:

(i) There exist extended monotonic additive value functions $\left(V_{j}\right)_{j=1}^{n}$ for $\geqslant$ on $X$ that are finite except possibly $V_{1}$ at minimal outcomes and $V_{n}$ at maximal outcomes, and that are continuous where they are finite. 
(ii) The binary relation $\succcurlyeq$ on $X$ is a continuous monotonic weak order that satisfies generalized triple cancellation.

The additive value functions in (i) are jointly cardinal on $\mathscr{C}_{\mathbf{r}}^{\boldsymbol{n}} \backslash\{$ extreme alternatives\}. If $n \geqq 3$ then generalized triple cancellation in Statement (ii) can be weakened to $C I$.

Concerning the uniqueness result at extreme alternatives, we shall see at the end of section 4.2 that $V_{n}$ must assign the value $\infty$ to maximal outcomes if that is the supremum of the values of $V_{n}$ over nonmaximal outcomes. If that supremum is not $\infty, V_{n}$ can either be taken to be that supremum, or $\propto$. Similarly, to minimal outcomes, $V_{1}$ either assigns the infimum over the nonminimal outcomes, or $-\infty$. The following theorem shows when the representation in Statement (i) above can be taken finite.

Theorem 3.3. We may add in Statement (i) of Theorem 3.2 that the additive value functions are everywhere finite (thus continuous) if we either:

(a) add in the theorem the assumption that there are no maximal or minimal outcomes,

or

(b) restrict the domain $X$ of $\geqslant$ to $\mathscr{C}_{r}^{n} \backslash\{$ extreme alternatives $\}$, instead of $\mathscr{C}_{r}^{n}$,

or

(c) add in Statement (ii) the second-order Archimedean axiom.

Each of these changes gives again equivalent Statements (i) and (ii).

Note that Theorem 3.3.(c) has given a characterization of additive representability on rank-ordered subsets in full generality. It shows that the lemma in the appendix of Green and Jullien (1988) can be corrected by addition of the second-order Archimedean axiom. The condition in the proposition below does not give a representation result in the strict sense because it is not directly in terms of the observable primitive, the preference relation.

Proposition 3.4. The additive value functions in Theorem 3.2(i) can be taken finite if and only if $V_{1}$ is bounded below on the nonminimal outcomes whenever a minimal outcome exists, and $V_{n}$ is bounded above on the nonmaximal outcomes whenever a maximal outcome exists.

Again, the proportionality condition below is not directly in terms of the preference relation. Wakker (1991, above Corollary 6) lists several conditions from the literature that characterize proportionality directly in terms of the preference relation. 
Proposition 3.5. The additive value functions in Theorem 3.2(i) can be taken finite if they are proportional on the set of nonminimal and nonmaximal outcomes.

The following corollary repeats some special cases.

Corollary 3.6. Suppose either $X=\mathbb{R}_{r}^{n}=\left\{\left(x_{1}, \ldots, x_{n}\right) \in \mathbb{R}^{n}: x_{1} \geqq \cdots \geqq x_{n}\right\}$, or $X=\left(\mathbb{R}_{++}\right)_{r}^{n}=\left\{\left(x_{1}, \ldots, x_{n}\right) \in\left(\mathbb{R}_{++}\right)^{n}: x_{1} \geqq \cdots \geqq x_{n}\right\} ; \geqslant$ on $X$ is an increasing continuous weak order that satisfies $C I$, and generalized triple cancellation if $n=2$. Then there exist jointly cardinal continuous increasing additive value functions.

If one sets $X=\left(\mathbb{R}_{+}\right)_{r}^{n}$ in the above corollary then, strictly speaking, it may happen that no additive value functions exist, because the additive value function $V_{1}$ may be 'driven to $-\infty$ ' at 0 . Note that the monotonicity conditions in the above results have ruled out inessential coordinates. Inessential coordinates can be suppressed from notation, thus do not complicate the above results. Definitions of the conditions in the Remark below are given in Wakker (1989b), as well as in many other references.

Remark 3.7. For $n=2$ generalized triple cancellation in the above results can be replaced by the 'Thomsen condition', or the 'hexagon condition'.

\subsection{Extreme alternatives and continuity}

Example 25a in the algebraic approach of Wakker (1991) showed that an additive value function $V_{n}$ at maximal outcomes, if finite, can take any value greater/equal the supremum over the nonmaximal outcomes. That was because no continuity was imposed. If continuity is imposed, such as in this paper, then the additive value function, if finite, simply has to be the mentioned supremum at maximal outcomes. Similar things apply to $V_{1}$ and minimal outcomes.

Example $3.8\left[V_{1}\right.$ driven to $\left.-\infty\right]$. See fig. 3. This is a topological version of Example 25b (fig. 4) in Wakker (1991). It satisfies all conditions in Theorem 3.2 , and shows that indeed an additive value function $V_{1}$ can be 'driven to $-\infty$ '. Similarly $V_{n}$ can be driven to $\infty$, see Example $25 \mathrm{c}$ in Wakker (1991). Continuity of $\geqslant$ and $\geqslant$ can also be derived for these examples; see also Lemmas 4.3 and $\Lambda .1$. Strictly speaking, these examples provide counter-examples to (nonextended) additive representation on rankordered subsets. 


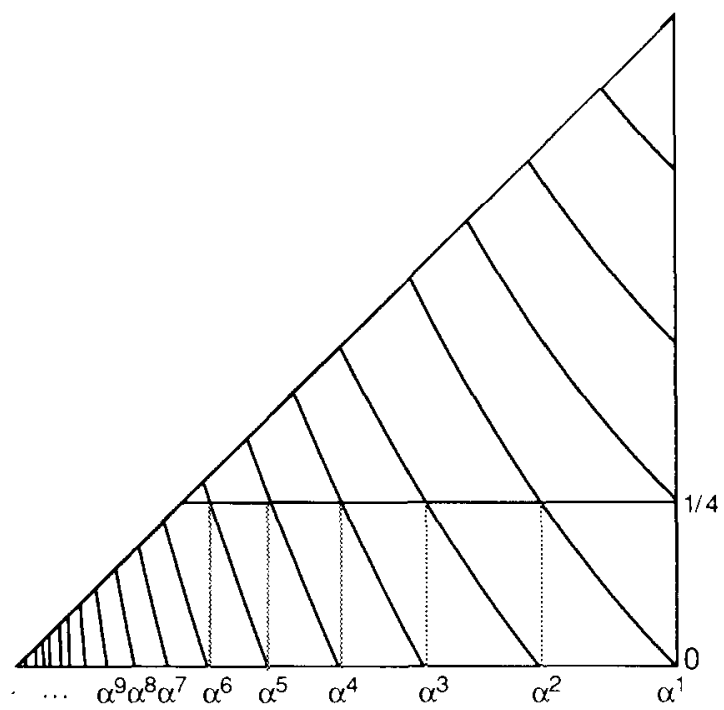

Fig. 3 [A counter-example to additive representation on a (rank-ordered) subset of a Cartesian product $]$. Let $n \geqq 2, \mathscr{C}=[0,1] ; \ni$ is defined on $\mathscr{C}_{r}^{n}$, and is represented by $\left(x_{1}, \ldots, x_{n}\right) \mapsto x_{1} \times \mathrm{e}^{x_{2}} \times$ $\cdots \times \mathrm{e}^{x_{n}}$. Any extended additive value functions $\left(W_{1}, \ldots, W_{n}\right)$ would be of the form $\left(\left[\tau_{1}+\sigma \ln \left(x_{1}\right)\right],\left[\tau_{2}+\sigma x_{2}\right], \ldots,\left[\tau_{n}+\sigma x_{n}\right]\right)$, with $\sigma>0$ and $\ln (0):=-\infty . W_{1}$ would satisfy, with $\alpha^{k}=\mathrm{e}^{(1-k) / 4}$ for all $k, W_{1}\left(\alpha^{k}\right)-W_{1}\left(\alpha^{k+1}\right)=W_{1}\left(\alpha^{k+1}\right)-W_{1}\left(\alpha^{k+2}\right)$. Thus $W_{1}$ would necessarily assign value $-\infty$ to 0 .

Let us now present two examples showing that, even on 'nice' subsets of Cartesian products, continuity of $\geqslant$ ' is problematic. In some cases it is not implied by the other conditions in the main theorems.

Example 3.9 [Absence of continuity of $\geqslant$ on $\mathscr{C}$ ]. Case 1a in fig. 4 shows that continuity of $\geqslant$ on $\mathscr{C}_{r}^{2} \backslash$ \{extreme alternatives $\}$ need not imply continuity of the associated binary relation $\succcurlyeq$ on $\mathscr{C}$. Similarly continuity of additive value functions on $\mathscr{C}_{r}^{2} \backslash\{$ extreme alternatives $\}$ is not implied by continuity of their sum. This example can be extended to dimension $n \geqq 3$ : Suppose again that $\mathscr{C}=] 0,1]$, endowed with the circle topology of fig. 4. For the topology $\mathscr{T}_{c}^{n}$ on the unit cube, any open set containing a point of the form $\left(1, \ldots, 1, x_{i+1}, \ldots, x_{n}\right) \quad\left(1>x_{i+1} \geqq \cdots \geqq x_{n}, i<n\right)$ contains cubes around all points of the form $\left(v_{1}, \ldots, v_{i}, x_{\mathrm{i}+1}, \ldots, x_{n}\right)$ for which, for all $j \leqq i, v_{j}=0$ or $v_{j}=1$. These cubes can be taken so small (diameters smaller than $\frac{1}{2} x_{i+1}$ ) that they intersect $\mathscr{C}_{r}^{n}$ only in the cube around $\left(1, \ldots, 1, x_{i+1}, \ldots, x_{n}\right)$. It straightforwardly follows that the restriction of $\mathscr{T}_{c}^{n}$ to $\mathscr{C}_{r}^{n} \backslash\{$ extreme alternatives coincides with the (restriction of) the Euclidean topology, and that $\left(x_{1}, \ldots, x_{n}\right) \mapsto x_{1}+\cdots+x_{n}$ is continuous and represents a continuous relation $\succcurlyeq$. The conclusions of the Cases $1 \mathrm{a}, 1 \mathrm{~b}$, and 2 in fig. 4 can be extended to dimension $n \geqq 3$. 


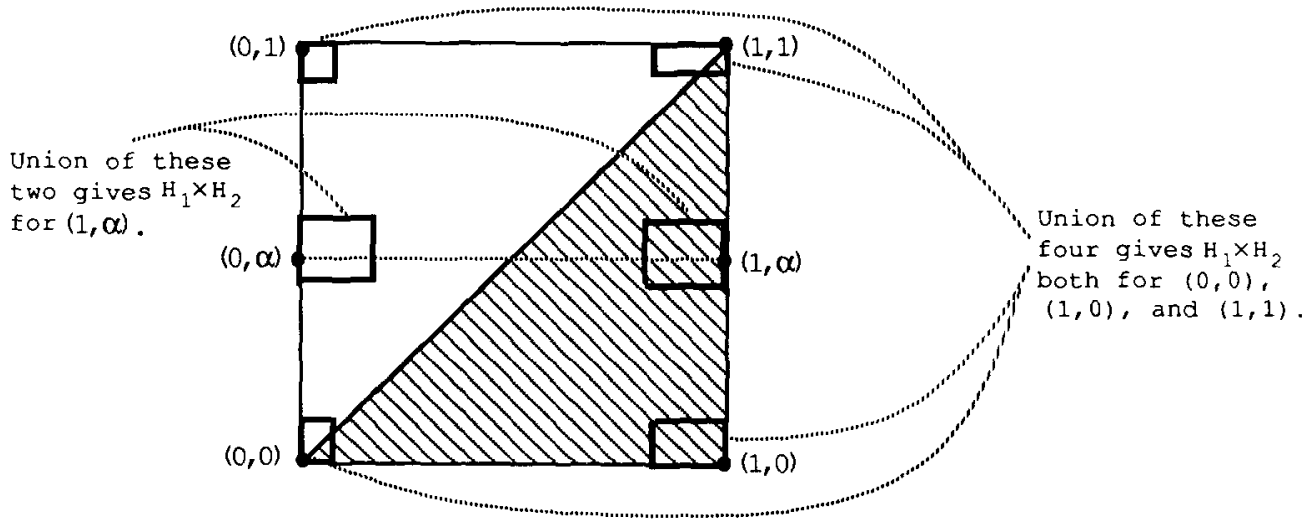

Fig. 4 [Topological complications on rank-ordered subsets (dashed in the figure)]. The circle topology $\mathscr{T}_{c}$ on $[0,1]$ is like the usual Euclidean topology with one exception: every open set containing 1 must contain 0 , and vice versa. Note that this is connected. Let us consider the closed unit square endowed with the product topology $\mathscr{T}_{c}^{2}$. For $0<\alpha<1$, any open neighborhood $H_{1} \times H_{2}$ of $(1, \alpha)$ must contain a rectangle around $(1, \alpha)$ as well as one around $(0, \alpha)$. An open neighborhood $H_{1} \times H_{2}$ of $(0,0)$ must contain a rectangle around each of the points $(0,0)$, $(1,0),(1,1),(0,1)$; this also holds for any open neighborhood of $(1,0),(1,1)$, as well as of $(0,1)$.

Now let us consider subsets of rank-ordered alternatives, and the restriction of $\mathscr{T}_{c}^{2}$. If none of the points $(0,0),(1,0),(1,1)$ is contained in the subset, then the restriction of $\mathscr{T}_{c}^{2}$ coincides with the usual Euclidean topology; this mainly follows from the observation that in the figure for any $0<\alpha<1$ one can take the rectangle around $(0, x)$ small enough not to intersect the dashed area; similarly open neighborhoods of points $(\alpha, 0)$ can be taken within the dashed area. If any of the points $(0,0),(1,0),(1,1)$ is contained, the topologies do not coincide.

In each of the cases below $\geqslant$ is represented by $\left(x_{1}, x_{2}\right) \mapsto x_{1}+x_{2}$. In each case $\mathscr{C}$ contains 1 , and $\succcurlyeq^{\prime}$ on $\mathscr{C}$ is not continuous: the set $\left\{\alpha \in \mathscr{C}: x>^{\prime} 1 / 2\right\}$ contains 1 but is not open because any open set containing 1 should contain an interval ]0, $\varepsilon$ for a small $\varepsilon>0$.

Case 1a. $\mathscr{C}=] 0,1]$, endowed with the restriction of the circle topology; $\geqslant$ is defined on $C_{r}^{2} \backslash\{(1,1)\}$. It is continuous because the topology on $\mathscr{C}_{r}^{2} \backslash\{(1,1)\}$ coincides with the usual Euclidean topology. This case also illustrates that on a subset of a Cartesian product an additive representation $\left[\left(x_{1}, x_{2}\right) \mapsto x_{1}+x_{2}\right]$ can be continuous whereas not all of the additive value functions are continuous: $x_{1} \mapsto x_{1}$ is not continuous as a function from $\left.] 0,1\right]$-with-the-circletopology to $\mathbb{R}$-with-the-usual-Euclidean-topology.

Case $l b . \mathscr{C}=] 0,1]$, endowed with the restriction of the circle topology, $\succcurlyeq$ is defined on $\mathscr{C}_{r}^{2}$; so now $(1,1)$ is included. Continuity of $\geqslant$ is violated because the set of alternatives strictly preferred to $(2 / 3,2 / 3)$ contains $(1,1)$ but does not contain $\{1\} \times] 0, \varepsilon[$ for some $\varepsilon>0$, as it should to be open.

Case 2. $\mathscr{C}=[0,1]$, endowed with the circle topology. The following reasoning shows discontinuity of $\geqslant$, both if it is defined on $\mathscr{L}_{r}^{2} \backslash\{(0,0),(1,1)\}$, and if it is extended to one or both of the alternatives $(0,0),(1,1)$. Any open set containing $(1,0)$ will contain alternatives of the form $(\beta, 0)$ for $\beta>0$ very small. Hence the set of alternatives strictly preferred to $(1 / 3,1 / 3)$ is not open, and $\geqslant$ is not continuous. 
Example 3.10 [Absence of continuity of $\geqslant$ on $\mathscr{C}$ ]. Suppose the Structural Assumption 3.1 holds, with the exception possibly of continuity of $\succcurlyeq$, further $n=2$, and $\mathscr{C}=[0,1]$ endowed with the usual Euclidean topology. $\mathscr{C}^{+}$, $\mathscr{C}^{-}$is any partition of $\mathscr{C}$, with both sets nonempty. Suppose $\geqslant^{\prime}$ has two equivalence classes $\mathscr{C}^{+}, \mathscr{C}^{-}$, with $\mathscr{C}^{+}$the preferred equivalence class. Obviously $\geqslant^{\prime}$ is not continuous. Set $V_{1}=0$ on $\mathscr{C}^{+}, V_{1}=-\infty$ on $\mathscr{C}^{-}, V_{2}=\infty$ on $\mathscr{C}^{+}, V_{2}=0$ on $\mathscr{C}^{-}$. Then both functions are constant, thus continuous, where they are finite.

(a) $X=\mathscr{C}_{r}^{2}$, as in Theorem 3.2 . $\geqslant$ is completely determined by monotonicy, has three equivalence classes, and by connectedness of $X$ cannot be continuous. Statement (i) of Theorem 3.2 however holds true. So (i) $\Rightarrow$ (ii) in Theorem 3.2 does not hold true. $\geqslant$ is a monotonic weak order and satisfies generalized triple cancellation as well as CI.

(b) $X=\mathscr{C}_{\text {r }}^{2} \backslash$ extreme alternatives $\}$, as in Theorem 3.3.(b). There is only one equivalence class, so that $\geqslant$ is continuous. All conditions of Statement (ii) of Theorem 3.2, in the version of Theorem 3.3.(b), are satisfied. Any $V_{1}, V_{2}$ are additive value functions. Obviously, they cannot be both monotonic and continuous.

Next we turn to positive results. We show that continuity of $\succcurlyeq^{\prime}$, while not in all cases, at least in most cases is implied by the other conditions; so in most cases it could have been omitted from the Structural Assumption 3.1.

Proposition 3.11. Suppose the Structural Assumption 3.1 holds, possibly without continuity of $\succcurlyeq$ on $\mathscr{C}$. Then $\succcurlyeq$ is continuous after all in each of the following, partly overlapping, cases:

(a) If there are no maximal outcomes, no minimal outcomes, and Statement (i) or Statement (ii) of Theorem 3.2 holds.

(b) If $X=\mathscr{C}_{r}^{n}$ and Statement (ii) of Theorem 3.2 holds true.

(c) If $X=\mathscr{C}_{r}^{n}$ and $n \geqq 3$, and Statement (i) or Statement (ii) of Theorem 3.2 holds true.

(d) If $X=\mathscr{C}_{r} \backslash\{$ extreme alternatives $\}, n \geqq 3$, and Statement (i) of Theorem 3.2 holds true.

So for $n \geqq 3$ Theorem 3.2 holds true without the assumption that $\succcurlyeq$ ' on $\mathscr{C}$ is continuous.

The results of Examples 3.9, 3.10, and Proposition 3.11 have been summarized in fig. 5 .

Less general, but more elegant, results could have been obtained by imposing in Statement (i) of Theorem 3.2 an extended continuity condition for extended functions, requiring that convergence to an argument with 


\begin{tabular}{|c|c|c|c|c|c|c|}
\hline & \multicolumn{3}{|c|}{$\begin{array}{l}\text { no maximal or } \\
\text { minimal outcomes }\end{array}$} & \multicolumn{3}{|c|}{$\begin{array}{l}\operatorname{maximal} \text { and/or } \\
\text { minimal outcomes }\end{array}$} \\
\hline \multirow[b]{3}{*}{$X=C_{r}^{n}$} & & $n=2$ & $n \geq 3$ & & $\mathrm{n}=2$ & $\mathrm{n} \geq 3$ \\
\hline & (i) & + & + & (i) & - & + \\
\hline & (ii) & + & + & (ii) & + & + \\
\hline & & $n=2$ & $\mathrm{n} \geq 3$ & & $\mathrm{n}=2$ & $n \geq 3$ \\
\hline$X=C_{r}^{M}$ & (i) & + & + & (i) & - & + \\
\hline \{extr.alts.\} & (ii) & + & + & (ii) & - & - \\
\hline
\end{tabular}

Fig. 5. + means that continuity of $\succcurlyeq$ is implied by the other conditions, - means it is not. This depends on the domain $X$ of $\geqslant$, the presence or absence of maximal/minimal outcomes, the dimension $n$, and whether Statement (i) or Statement (ii) in Theorem 3.2 is assumed to hold.

function-value $\infty$ or $-\infty$ implies convergence of function-values to $\infty$ or $-\infty$, respectively. Then joint cardinality would have resulted on the entire domain in all results, and Example 3.10 would have been ruled out. We do not pursue this line.

\section{Proofs}

\subsection{No extreme alternatives: Proof of Theorem 3.3.(b), thus of Theorem 3.3.(a)}

We assume throughout this subsection that Assumption 3.1 holds, and that $X=\mathscr{C}_{r}^{n} \backslash\{$ extreme alternatives $\}$. First suppose (i) in Theorem 3.2 holds. Note that, by the definition of additive value functions, $V_{1}$ only has to be defined on the nonminimal outcomes, $V_{n}$ only on the nonmaximal outcomes. Continuity of each $V_{i}$ implies continuity of each $V_{i}^{\prime}:\left(x_{1}, \ldots, x_{n}\right) \mapsto V_{i}\left(x_{i}\right)$ (also for $i=1$ and $i=n$ ), thus of the sum of these functions. This implies continuity of $\geqslant$. The other conditions of (ii) are straightforward.

Next we assume (ii) holds (with generalized triple cancellation weakened to CI for $n \geqq 3$ ), and derive (i). We derive this as a corollary from Theorem 4.(b) in Wakker (1991). There the following two conditions were used instead of continuity to derive additive representability; the two conditions will now be derived from continuity. Finally we shall then establish continuity of the 
additive value functions, and the uniqueness result. $\succcurlyeq$ satisfies restricted solvability on $E$ if, for all $x_{-i} x, y, x_{-i} \gamma \in E$ :

$$
x_{-i} \alpha>y>x_{-i} \gamma \quad \Rightarrow \quad \exists \beta \in \mathscr{C}: x_{-i} \beta \in E, x_{-i} \beta \sim y .
$$

If $\geqslant^{\prime}$ is trivial (has one equivalence class) then, all alternatives being both maximal and minimal, $X$ is empty, so Statement (i) is vacuous. So we suppose henceforth that $\succcurlyeq^{\prime}$ has at least two equivalence classes.

Lemma 4.1. $\succcurlyeq$ satisfies restricted solvability.

Proof. Suppose $x_{-i} \alpha \succ y>x_{-i} \gamma$. By monotonicity, $\alpha \succ^{\prime} \gamma$. We first derive openness of the set $E^{+}:=\left\{\sigma \in \mathscr{C}: \sigma \geqslant^{\prime} \alpha\right.$ or $\left(\alpha \succ^{\prime} \sigma \succ^{\prime} \gamma\right.$ and $\left.\left.x_{-i} \sigma \succ y\right)\right\}$. Take any $\sigma \in E^{+}$. We find an open neighborhood $H$ of $\sigma$ within $E^{+}$. If $\sigma \succ^{\prime} \alpha$ then, by continuity of $\succcurlyeq^{\prime}, H=\left\{\tau \in \mathscr{C}: \tau \succ^{\prime} \alpha\right\}$ can be taken. So say $\sigma \preccurlyeq^{\prime} \alpha$. Then, both if $\sigma \sim^{\prime} \alpha$ and if $\sigma\left\langle^{\prime} \alpha, x_{-i} \sigma \in X\right.$ and $x_{-i} \sigma \succ y$. By continuity of $\geqslant$ with respect to the restriction of the product topology, there exists an open neighborhood $\left(H_{1} \times \cdots \times H_{n}\right) \cap X$ of $x_{-i} \sigma$ with $H_{j} \subset \mathscr{C}$ open for all $j$, all elements of which are strictly preferred to $y$. Obviously, not $\gamma \in H_{i}$ because $x_{-i} \gamma$ is in $X$ but is strictly dispreferred to $y$. There may however be outcomes $\tau \prec^{\prime} \gamma$ in $H_{i}$, simply because $x_{-i} \tau \notin X$, i.e., $\tau \prec^{\prime} x_{i+1}$. We exclude such $\tau$ by considering, instead of $H_{i}, H:=H_{i} \cap\left\{\tau \in \mathscr{C}: \tau \succ^{\prime} \gamma\right\}$, which by continuity of $\geqslant$ is open, and contains $\sigma$. So we may, and do, assume that $H \subset\left\{\tau \in \mathscr{C}: \tau \succ^{\prime} \gamma\right\}$. To see that $H \subset E^{+}$, note that for each $\tau \in H$ either $\tau \geqslant{ }^{\prime} \alpha$ or $\tau<^{\prime} \alpha$ in which case indeed $\alpha \succ^{\prime} \tau \succ^{\prime} \gamma$ and $x_{-i} \tau \in\left(H_{1} \times \cdots \times H_{n}\right) \cap X$, so $x_{-i} \tau \succ y$.

Analogously, the set $E^{-}:=\left\{\sigma \in \mathscr{C}: \sigma \leqslant^{\prime} \gamma\right.$ or $\left(\alpha \succ^{\prime} \sigma \succ^{\prime} \gamma\right.$ and $\left.\left.x_{-i} \sigma \prec y\right)\right\}$ is open. Both $E^{+}$and $E^{-}$are nonempty, and they are disjoint. By connectedness of $\mathscr{C}$, there must be a $\beta$ contained neither in $E^{+}$nor in $E^{-}$. Because $\alpha \succ^{\prime} \beta \succ^{\prime} \gamma$, we have $x_{-i} \beta \in X ; x_{-i} \beta \sim y$ follows.

Lemma 4.2. $\succcurlyeq$ satisfies the Archimedean axiom.

Proof. Suppose there is an infinite standard sequence $\alpha^{1}, \alpha^{2}, \ldots$ on coordinate $j$. I.e., there exist $x, i \neq j$, and $v_{i} \chi^{\prime} w_{i} \in \mathscr{C}$, such that $x_{-i, j} v_{i}, \alpha^{k} \sim$ $x_{-i, j} w_{i}, \alpha^{k+1}$ for all $k=1,2, \ldots$, with further all alternatives in question contained in $X$. Suppose the standard sequence is bounded, i.e., there exist $\alpha^{\text {sup }}$ and $\alpha^{\text {inf }}$ that are $j$ th coordinates of elements of $X$, such that $\alpha^{\text {sup }} \succcurlyeq^{\prime} \alpha^{k} \succcurlyeq^{\prime} \alpha^{\text {inf }}$ for all $k$; note that $\alpha^{\text {inf }}$ is nonminimal if $j=1, \alpha^{\text {sup }}$ is nonmaximal if $j=n$. Contradiction will follow.

Case (a). $\quad v_{i} \succ^{\prime} w_{i}$. By monotonicity, $\alpha^{k+1} \succ^{\prime} \alpha^{k}$ for all $k$. Let $E^{+}:=\{\beta \in \mathscr{C}$ : $\left.\forall k: \beta \geqslant \alpha^{k}\right\}, E^{-}:=\left\{\beta \in \mathscr{C}: \exists k: \beta \prec^{\prime} \alpha^{k}\right\}$. By continuity of $\succcurlyeq^{\prime}, E^{-}$is a union of 
open sets, so is again open. Further $E^{-}$and $E^{+}$are disjoint, nonempty, and their union is $\mathscr{C}$. Thus, by connectedness of $\mathscr{C}, E^{+}$cannot be open. To establish contradiction, we finally derive openness of $E^{+}$. Suppose $\mu^{1} \geqslant \alpha^{k}$ for all $k$. It suffices, by continuity of $\geqslant^{\prime}$, to find a $\mu^{0}<^{\prime} \mu^{1}$ such that still $\forall k: \mu^{0} \geqslant \alpha^{k}$.

If $x_{-i, j} w_{i}, \mu^{1} \notin X$ then $j>1$ and $\mu^{1} \succ^{\prime} x_{j-1} \geqslant^{\prime} \alpha^{k}$ (with $x_{j-1}:=w_{i}$ if $i=j-1$ ) for all $k$, the latter preference because $x_{-i, j} w_{i}, \alpha^{k}$ is rank-ordered for all $k$. Then $\mu^{0}=x_{j-1}$ can be taken. A similar reasoning applies if $x_{-i, j} v_{i}, \mu^{1} \notin X$. So suppose $x_{-i, j} w_{i}, \mu^{1} \in X$ and $x_{-i, j} v_{i}, \mu^{1} \in X$. By monotonicity, for all $k$,

$$
x_{-i, j} v_{i}, \mu^{1}>x_{-i, j} w_{i}, \mu^{1} \succcurlyeq x_{-i, j} w_{i}, \alpha^{k+2}>x_{-i, j} w_{i}, \alpha^{k+1} \sim x_{-i, j} v_{i}, \alpha^{k}
$$

By restricted solvability, there exists a $\mu^{0}$ between $\mu^{1}$ and $\alpha^{1}$ (or any other $\alpha^{k}$ ) such that $x_{-i, j} v_{i}, \mu^{0} \sim x_{-i, j} w_{i}, \mu^{1}$. We saw above that, for each $k$, the latter is strictly preferred to $x_{-i, j} v_{i}, \alpha^{k}$, so $x_{-i, j} v_{i}, \mu^{0}>x_{-i, j} v_{i}, \alpha^{k}$. By monotonicity $\mu^{0} \succ^{\prime} \alpha^{k}$ for all $k$, and $\mu^{0}$ is as desired.

Case (b). $\quad v_{i} \prec^{\prime} w_{i}$. This is analogous to the above case. Now $\alpha^{k+1} \prec^{\prime} \alpha^{k}$ for all $k$, and one defines $E^{+}:=\left\{\beta \in \mathscr{C}: \exists k: \beta \succ^{\prime} \alpha^{k}\right\}, E^{-}:=\left\{\beta \in \mathscr{C}: \forall k: \beta \aleph^{\prime} \alpha^{k}\right\}$, etc.

So $\geqslant$ is a weak order that satisfies restricted solvability, the Archimedean axiom, monotonicity, CI, and generalized triple cancellation if $n=2$. Theorem 4.(b) of Wakker (1991) implies that there exist additive value functions $\left(V_{j}\right)_{j=1}^{n}$ for $\geqslant$. Continuity follows from Lemma A.1 in the Appendix.

Finally we establish the uniqueness result: Joint cardinality of the additive value functions is implied by cardinality of the additive representation, as implied by Theorem 4.(b) in Wakker (1991). For the application of the latter result note that, because $\succcurlyeq$ is continuous with respect to a connected topology, as soon as it has two or more equivalence classes, it must have infinitely many equivalence classes.

\subsection{Extreme alternative: Proof of Theorem 3.2}

As a preparation we show that the requirement of continuity of the preference relation at extreme outcomes is immaterial.

Lemma 4.3. Suppose that the Structural Assumption 3.1 holds with $X=\mathscr{C}_{r}^{n}$, and that $\succcurlyeq$ is a monotonic weak order that is continuous on $\mathscr{C}_{\text {r }}^{n} \backslash\{$ extreme alternatives $\}$. Then $\succcurlyeq$ is continuous on $\mathscr{C}_{r}^{n}$.

Proof. We derive openness of the set $P^{\prime}(y):=\left\{x \in \mathscr{C}_{r}^{n}: x>y\right\}$. If $y$ is maximal then $P^{\prime}(y)$ is empty, thus open. So assume $y$ is not maximal. 
$P^{\prime}(y) \backslash\{$ maximal alternatives $\}=: P(y)=\left\{x \in \mathscr{C}_{r}^{n} \backslash\{\right.$ extreme alternatives $\left.\}: x>\mathrm{y}\right\}$ is an open subset of $\mathscr{G}_{r}^{\backslash} \backslash\{$ extreme alternatives $\}$. So there exists an open set $P \subset \mathscr{C}^{n}$ intersecting $C_{r}^{n} \backslash\{$ extreme alternatives $\}$ in exactly that subset. By monotonicity we may assume that $P \subset\left(\bigcup_{j=1}^{n}\left\{x \in \mathscr{C}^{n}: x_{j}\right.\right.$ nonminimal $\left.\}\right)$, by intersecting with that latter open set. I.e., we may assume that $P$ does not contain minimal alternatives. If no maximal outcomes exist then $P^{\prime}(y)=$ $P \cap \mathscr{C}_{r}^{n}$ and we are done. So suppose there exist maximal outcomes. We show that $P$ may be assumed to contain maximal alternatives.

To that end we first find an alternative $\tilde{y} \in P^{\prime}(y)$ with all coordinates nonmaximal. Because $y$ is nonmaximal, there is a coordinate $i \leqq n$ such that $y_{1}, \ldots, y_{i-1}$ are maximal and $y_{i}$ is nonmaximal. Say $i \geqq 2$, otherwise $\tilde{y}=y$ can be taken. By continuity of $\geqslant^{\prime}$ and connectedness of $\mathscr{C}$ there exists a nonmaximal $\tilde{y}_{i} \succ^{\prime} y_{i}$. By monotonicity, the nonmaximal $y_{-i} \tilde{y}_{i} \in P(y)$. There must exist open $A_{1}, \ldots, A_{n}$ such that $y_{-i} \tilde{y}_{i} \in A_{1} \times \cdots \times A_{n} \subset P$. We may, and do, replace $A_{i-1}$ by the open nonempty $A_{i-1} \cap\left\{\gamma: \gamma \succ \tilde{y}_{i}\right\}$. By closedness of \{maximal outcomes $\} \subset \mathscr{C}$, and connectedness of $\mathscr{C}, A_{i}{ }_{1}$ contains a nonmaximal outcome $\tilde{y}_{i-1}$. Then $y_{-i},(i-1) \tilde{y}_{i}, \tilde{y}_{i-1} \in P(y)$ : we have replaced the maximal $y_{i-1}$ by a nonmaximal $\tilde{y}_{i-1}$ without leaving $P(y)$. Continuing that way we end up with $\tilde{y} \in P(y)$ that has all coordinates nonmaximal.

The set $\bigcap_{i=1}^{n}\left\{v \in \mathscr{C}^{n}: v_{i} \succ^{\prime} \tilde{y}_{i}\right\}$ is open. All of its elements being strictly preferred to $\tilde{y}$, its intersection with $\mathscr{C}_{r}^{n}$ is a subset of $P(y)$, and we may replace $P$ by its union with $\bigcap_{i=1}^{n}\left\{v \in \mathscr{C}^{n}: v_{i} \succ^{\prime} \tilde{y}_{i}\right\}$. This contains all maximal alternatives; openness of $P^{\prime}(y)$ follows. Analogously, $\left\{x \in \mathscr{C}_{r}^{n}: x \prec y\right\}$ is open.

We next derive the implication (i) $\Rightarrow$ (ii) in Theorem 3.2. Transitivity, completeness, and monotonicity are direct. Generalized triple cancellation, hence CI, follows straightforwardly, either by elimination of cases, or by substitution of the extended additive representation. Next we turn to continuity. If we restrict both the additive function and the preference relation to the nonextreme alternatives, then these restrictions are all continuous, as well as the associated additive value functions. Now continuity of $\geqslant$ follows from Lemma 4.3. It is a remarkable property of rankordered sets that continuity of the additive value functions on the nonextreme alternatives implies continuity of $\geqslant$ on all alternatives.

For (ii) $\Rightarrow$ (i), suppose $\geqslant$ contains at least two, so infinitely many, equivalence classes, to avoid triviality. Theorem 3.3.(b) gives an additive representation $V$ on $\mathscr{C}_{r}^{n} \backslash\{$ extreme alternatives $\}$. We can assign to maximal extreme alternatives any $V$-value greater/equal the supremum over the other alternatives, by letting $V_{n}$ assign to the maximal outcome any value greater/equal $\sup \left(V_{n}(\{\right.$ nonmaximal outcomes $\left.\})\right)$. Note that that supremum is not assigned to any nonmaximal outcome, $\geqslant$ ' being continuous with respect to a connected topology. The supremum may be $\infty$. For continuity of $V_{n}$ 
where it is finite, the only finite value that $V_{n}$ can assign to a maximal outcome is the mentioned supremum. The case of minimal extreme alternatives is similar, let $V_{1}$ (minimal outcome $)=\inf (U\{$ nonminimal outcomes $\left.\})\right)$ or $-\infty$.

\subsection{The second-order Archimedean axiom: Proof of Theorem 3.3.(c)}

Below the definition of the second-order Archimedean axiom it was already pointed out that the condition is necessary for real-valued additive representability. So let us assume that it holds, together with Statement (i), thus (ii), of Theorem 3.2. Suppose that there exists a maximal outcome with $V_{n}$-value 'driven to $\infty$ ', i.e., $\sup \left\{V_{n}(\mathscr{C} \backslash\{\right.$ maximal outcomes $\})=\infty$. The case of $V_{1}$ driven to $-\infty$ is similar. Violation of the second-order Archimedean axiom will be derived. We construct an infinite increasing second-order standard sequence $\left(\alpha^{k}\right)_{k=1}^{\infty}$, obviously bounded above by the maximal outcomes, and bounded below by $\alpha^{1}$. Take any $\left(\alpha^{k}\right)_{k=1}^{\infty}$ with $V_{n}\left(\alpha^{k+1}\right)-V_{n}\left(\alpha^{k}\right)=1$ for all $k . V_{n}(\mathscr{C} \backslash\{$ maximal outcomes $\})$ being connected, this is possible. Fix $k$. Because $V_{n-1}\left(\left\{\alpha \in \mathscr{C}: \alpha^{k+2} \preccurlyeq^{\prime} \alpha \preccurlyeq^{\prime} \alpha^{k+3}\right\}\right)$ is a nondegenerate interval, we can find $\alpha^{k+2} \leqslant^{\prime} \gamma \prec^{\prime} \delta \leqslant \alpha^{k+3}$ and $l \in \mathbb{N}$ such that $V_{n-1}(\delta)-V_{n-1}(\gamma)=1 / l$. Next we take a sequence $\left(\beta^{m}\right)_{m=0}^{2 l}$ such that $\beta^{0}=\alpha^{k}, \beta^{l}=\alpha^{k+1}, \beta^{2 l}=\alpha^{k+2}$, and $V_{n}\left(\beta^{m+1}\right)-V_{n}\left(\beta^{m}\right)=1 / l$ for all $0 \leqq m \leqq 2 l-1$. Then, with $z_{1}=\cdots=z_{n-2}:=\alpha^{k+3}$ to ensure rank-orderedness of the following alternatives, $z_{-(n-1), n} \gamma, \beta^{m+1} \sim$ $z_{-(n-1), n} \delta, \beta^{m}$ for all $0 \leqq m \leqq 2 l-1$. In other words, $\left(\beta^{m}\right)_{k=0}^{2 l}$ is a standard sequence on coordinate $n$. As this can be obtained for each $k,\left(\alpha^{k}\right)_{k=1}^{\infty}$ is an infinite bounded second-order standard sequence. A violation of the secondorder Archimedean axiom has been obtained.

\subsection{Proof of Propositions 3.4 and 3.5, Corollary 3.6, Remark 3.7, and Proposition 3.11}

Proposition 3.4 follows immediately from section 4.2, or from Proposition 5 in Wakker (1991); restricted solvability, as required in the latter, is implied by Lemma 4.1. Proposition 3.5 is a corollary of Proposition 3.4, or of Corollary 6 in Wakker (1991) and again Lemma 4.1. Corollary 3.6 is a special case of Theorem 3.3.(a). Remark 3.7 is analogous to Remark 23 in Wakker (1991). Note that in the topological approach, contrary to the algebraic approach, also the hexagon condition can be used to obtain additive representability for two dimensions; see for instance Wakker (1989b, Theorem III.6.6(ii)).

In Proposition 3.11, Statement (a) follows from continuity of $V_{1}$ (representing $\geqslant$ ' by monotonicity) on $\mathscr{C}$, and Statement (b), proved next.

Under the conditions of (b) we derive openness of $\left\{\beta \in \mathscr{C}: \beta \succ^{\prime} \alpha\right\}$. Suppose $\gamma \succ^{\prime} \alpha$. There exist open $A_{1}, \ldots, A_{n} \subset \mathscr{C}$ such that $(\gamma, \ldots, \gamma) \in$ 
$\left(A_{1} \times \cdots \times A_{n} \cap X\right) \subset\{x \in X: \quad x>(\alpha, \ldots, \alpha)\}$, the latter set being open. $A_{1} \cap \cdots \cap A_{n}$ gives an open neighborhood of $\gamma$ within $\left\{\beta \in \mathscr{C}: \beta \succ^{\prime} \alpha\right\}$. The latter set is open. Similarly $\left\{\beta \in \mathscr{C}: \beta \prec^{\prime} \alpha\right\}$ is open, and $\succcurlyeq^{\prime}$ is continuous.

Under the conditions of Statement (c), continuity of $\geqslant$ ' follows either from Statement (i) of Theorem 3.2 and monotonicity and continuity of $V_{2}$, or from Statement (b).

The conditions of Statement (d) imply continuity of $\geqslant$ by monotonicity and continuity of $V_{2}$.

\section{Conclusion}

The additive representation of preferences on subsets of Cartesian products is more complicated than has usually been thought. Several incorrect ideas have been accepted almost as common knowledge. To prevent future misunderstandings we have described and criticized them. Further we have given positive results for the special and simple, but presently most important, case: the rank-ordered cone. Still the proofs were not simple. Maximal and minimal alternatives cause complications. The obtained result will make possible the derivation of further rcsults for rank-dependent approaches to decision making under risk and uncertainty, and the derivation of further results for generalizations of the Gini index for welfare theory.

\section{Appendix A. Continuity of additive functions on rank-ordered sets}

The following lemma, used in the proof of Theorem 3.3.(b), may have interest of its own in adapting Theorem 3.1 of Wakker (1988) to rankordered sets. Hence we repeat all assumptions. Monotonicity is implied below by the requirement that all $V_{j}^{\prime}$ s represent $\succcurlyeq^{\prime}$ on their domain.

Lemma A.1. Suppose the Structural Assumption 3.1 holds, with $X=$ $\mathscr{C}_{r}^{n} \backslash\{$ extreme alternatives $\}, V_{2}, \ldots, V_{n-1}: \mathscr{C} \rightarrow \mathbb{R}, V_{1}: \mathscr{C} \backslash\{$ minimal outcomes $\} \rightarrow \mathbb{R}$, $V_{n}: \mathscr{C} \backslash\{$ maximal outcomes $\} \rightarrow \mathbb{R}$, where all these functions represent $\geqslant$ on their domain and are nonconstant. Suppose $\succcurlyeq$ on $X$, represented by $\left(x_{1}, \ldots, x_{n}\right) \mapsto$ $\sum_{j=1}^{n} V_{j}\left(x_{j}\right)$, is continuous. Then $V_{1}, \ldots, V_{n}$ are continuous.

Proof. Suppose some $V_{j}$ is not continuous. Then it certainly is not continuous with respect to the order topology induced by $\geqslant$ '. Contradiction will follow. The domain of $V_{j}$ is connected with respect to the order topology, also for $j=1$ and $j=n$. For $j=1$ and $j=n$ this follows by standard techniques, see for instance Wakker (1989b, Lemma VI.7.4). Hence, again by standard reasonings, there must exist a 'gap' of the form $] \mu, v]$ or of the form $\left[\mu, v\left[\right.\right.$ in the range of $V_{j}$, say the latter. More precisely, $v$ is in the range of $V_{j}, \mu$ is not, neither is any element between the two. But there is a 
sequence of elements in the range of $V_{j}$ converging to $\mu$ from below. Let $V_{j}(\tilde{v})=v$. We distinguish two cases.

Case 1. $j<n$. We first show that

$$
v-\mu>V_{j+1}\left(x_{j+1}\right)-V_{j+1}\left(y_{j+1}\right)>0
$$

for no $x_{j+1}, y_{j+1} \in V_{j}^{-1}(]-\infty, \mu[)$. Suppose there were such $x_{j+1}, y_{j+1}$. Contradiction will follow. Fix $z_{1}=\cdots=z_{j}=\tilde{v}, z_{j+1}=\cdots=z_{n}=y_{j+1}$. These choices imply that the alternatives below are indeed rank-ordered.

By $V_{j+1}\left(x_{j+1}\right)-V_{j+1}\left(y_{j+1}\right)<v-\mu<V_{j}(\tilde{v})-V_{j}\left(x_{j+1}\right)$ and monotonicity we get:

$$
z_{-j,(j+1)} x_{j+1}, x_{j+1}<z_{-j,(j+1)} \tilde{v}, y_{j+1}<z_{-j,(j+1)} \tilde{v}, x_{j+1} .
$$

By restricted solvability, as implied by Lemma 4.1 , there exists $b_{j}$ between $x_{j+1}$ and $\tilde{v}$ such that $z_{-j,(j+1)} b_{j}, x_{j+1} \sim z_{-j,(j+1)} \tilde{v}, y_{j+1}$. This would however imply $v>V_{j}\left(b_{j}\right)>\mu$, in contradiction with the assumed gap. So (A.1) holds.

The assumption of the gap implies that there are at least countably many equivalence classes of outcomes with $V_{j}$-value below $\mu$, so certainly below $\tilde{v}$. Actually, as soon as there exist two, then by standard reasonings there are uncourtably many; see for instance Wakker (1989b, Lemma VI.7.4 and p. 42, lines 3/4). By (A.1) that would generate uncountably many mutually disjoint open intervals with length $v-\mu$ and midpoints $V_{j+1}\left(a_{j+1}\right)$ for $a_{j+1} \preccurlyeq^{\prime} \tilde{v}$. This is impossible by denseness of the rationals in the reals. Contradiction has resulted.

Case 2. $j=n$. Now $\tilde{v}$ cannot be maximal, because maximal outcomes are not contained in the domain of $V_{n}$. Analogously to Case 1 above, we can show that $0<V_{j-1}\left(x_{j-1}\right)-V_{j-1}\left(y_{j-1}\right)<v-\mu$ for no $x_{j-1}, y_{j-1}$ from $V_{j}^{1}(] v, \infty[)$. Again, there are uncountably many equivalence classes in $V_{j}^{-1}(] v, \infty[)$. This would induce uncountably many mutually disjoint intervals within $\mathbb{R}$, which cannot be. Contradiction has resulted.

The following remark, formulated somewhat informally, is straightforward.

Remark A.2. Suppose the extreme outcomes are also included in the domains of $V_{1}, V_{n}$. If then $V_{1}(\mathscr{C} \backslash\{$ minimal outcomes $\})$ is unbounded from below, $V_{1}$ is $-\infty$ at minimal outcomes. Otherwise $V_{1}$ can be redefined as the infimum of $V_{1}(\mathscr{C} \backslash$ minimal outcomes\}) at minimal outcomes without affecting the represented $\geqslant$, and then is continuous. If $V_{n}(\mathscr{C} \backslash$ \{maximal outcomes\}) is unbounded from above, then $V_{n}$ is $\infty$ at maximal outcomes. Otherwise $V_{n}$ can be redefined as the supremum of $V_{n}(\mathscr{C} \backslash$ \{maximal outcomes $\left.\}\right)$ at maximal outcomes, and then is continuous. 


\section{References}

Aczél, J., 1966, Lectures on functional equations and their applications (Academic Press, New York).

Becker, J.L. and R.K. Sarin, 1987, Lottery dependent utility, Management Science 33, $1367-1382$.

Birnbaum, M.H., 1974, The nonadditivity of personal impressions, Journal of Experimental Psychology 102, 543-561.

Birnbaum, M.H. and S.E. Sutton, 1990, Scale convergence and utility measurement, Organizational behavior and human decision processes, forthcoming.

Blaschke, W. and G. Bol, 1938, Geometrie der Gewebe (Springer, Berlin).

Chew, S.H., 1989, Axiomatic utility theories with the betweenness property, Annals of Operations Research 19, 273-298.

Chew, S.H. and L.G. Epstein, 1989, A unifying approach to axiomatic non-expccted utility theories, Journal of Economic Theory 49, 207-240.

Chew, S.H. and P.P. Wakker, 1991, Generalizing choquet expected utility by weakening Savage's sure-thing principle, in preparation.

Debreu, G., 1960, Topological methods in cardinal utility theory, in: K.J. Arrow, S. Karlin and P. Suppes, eds. 1959, Mathematical methods in the social sciences (Stanford University Press, Stanford, CA) 16-26.

Debreu, G., 1990, Personal communication.

Doignon, J.P. and J.C. Falmagne, 1974, Difference measurement and simple scalability with restricted solvability, Journal of Mathematical Psychology 11, 473-499.

Ebert, U., 1988a, Measurement of inequality: An attempt at unification and generalization, Social Choice and Welfare 5, 147-169.

Ebert, U., 1988b, Rawls and Bentham reconciled, Theory and Decision 24, 215-223.

Fishburn, P.C., 1970, Utility theory for decision making (Wiley, New York).

Fishburn, P.C., 1976, Utility independence on subsets of product sets, Operations Research 24, 245-255.

Gilboa, I., 1988, A combination of expected utility theory and maxmin decision criteria, Journal of Mathematical Psychology, 32, 405-420.

Gorman, W.M., 1968, The structure of utility functions, Review of Economic Studies 35, $367-390$.

Green, J. and B. Jullien, 1988, Ordinal independence in non-linear utility theory, Journal of Risk and Uncertainty 1, 355-387, and erratum, 2 (1989) 119.

Hosszù, M., 1964, On local solutions of the generalized functional equation of associativity, Annales Universitatis Scientia Budapest Eötvõs Loránd Sectio Math. 7, 129-132.

Jaffray, J.Y., 1974a, Existence, propriétés de continuité, additivité de fonctions d'utilité sur un espace partiellement ou totalement ordonné. Ph.D. dissertation (Université de Paris VI, Paris).

Jaffray, J.Y., 1974b, On the extension of additive utilities to infinite sets, Journal of Mathematical Psychology 11, 431-452.

Jaffray, J.Y., 1988, Choice under risk and the security factor, Theory and Decision 24, 169-200.

Kahneman, D. and A. Tversky, 1979, Prospect theory: An analysis of decision under risk, Econometrica 47, 263-291.

Krantz, D.H., R.D. Luce, P. Suppes and A. Tversky, 1971, Foundations of measurement, Vol I, Additive and polynomial representations (Academic Press, New York).

Luce, R.D., D.H. Krantz, P. Suppes and A. Tversky, 1990, Foundations of measurement, Vol. III, Representation, axiomatization, and invariance (Academic Press, New York).

Miyamoto, J.M., 1988, Generic utility theory: Measurement foundations and applications in multiattribute utility theory, Journal of Mathematical Psychology 32, 357-404.

Nakamura, Y., 1990, Subjective expected utility with non-additive probabilities on finite state spaces, Journal of Economic Theory 51, 346-366.

Quiggin, J., 1982, A theory of anticipated utility, Journal of Economic Behaviour and Organization 3, 323-343.

Quiggin, J., 1989, Sure things - dominance and independence rules for choice under uncertainty, Annals of Operations Research 19, 335-357. 
Radó, F., 1959, Équations fonctionnelles caractérisant les nomogrammes avec trois échelles rectilignes, Mathematica Universitatae Cluj 1, 143-166.

Schmeidler, D., 1989, Subjective probability and expected utility without additivitiy, Econometrica 57, 571-587.

Segal, U., 1990, Additively separable representations on non-convex sets, manuscript (Department of Economics, University of Toronto, Toronto, Ont.).

Stengel, B. von, 1990, Closure properties of independence concepts for continuous utilities, Mathematics of Operations Research, accepted under condition of revision.

Wakker, P.P., 1986, Representations of choice situations, Ph.D. dissertation (Department of Economics, University of Brabant, Tilburg, the Netherlands).

Wakker, P.P., 1988, The algebraic versus the topological approach to additive representations, Journal of Mathematical Psychology 32, 421-435.

Wakker, P.P., 1989a, Continuous subjective expected utility with nonadditive probabilities, Journal of Mathematical Economics 18, 1-27.

Wakker, P.P., 1989b, Additive representations of preferences, A new foundation of decision analysis (Kluwer Academic Publishers, Dordrecht).

Wakker, P.P., 1990, From local to global additive representation (Duke University, Fuqua School of Business).

Wakker, P.P., 1991, Additive representations on rank-ordered sets. I. The algebraic approach, Journal of Mathematical Psychology 35, 501-531. 